\title{
The BYPASS-CTCA Study: the value of Computed Tomography Cardiac Angiography (CTCA) in improving patient-related outcomes in patients with previous bypass operation undergoing invasive coronary angiography: Study Protocol of a Randomised Controlled Trial
}

\author{
Anne-Marie Beirne ${ }^{1,2,3}$, Krishnaraj S. Rathod ${ }^{1,2,3}$, Emily Castle ${ }^{4}$, Mervyn Andiapen $^{1,2,3}$, Amy Richards ${ }^{2,3}$, \\ Anna Bellin ${ }^{5}$, Victoria Hammond ${ }^{5}$, Thomas Godec ${ }^{5}$, James C. Moon ${ }^{3,4}$, Ceri Davies ${ }^{3,4}$, \\ Christos V. Bourantas ${ }^{2,3}$, Andrew Wragg ${ }^{2,3}$, Amrita Ahluwalia ${ }^{1,3,5}$, Francesca Pugliese ${ }^{3,4}$, \\ Anthony Mathur ${ }^{1,2,3 \#}$, Daniel A. Jones ${ }^{1,2,3,5 \#}$ \\ ${ }^{1}$ Centre for Cardiovascular Medicine and Devices, William Harvey Research Institute, Queen Mary University of London, London, UK; ${ }^{2}$ Barts \\ Interventional Group, Barts Heart Centre, Barts Health NHS Trust, London, UK; ${ }^{3}$ NIHR Barts Biomedical Research Centre, Barts Heart Centre \\ and William Harvey Research Institute, Queen Mary University of London, London, UK; ${ }^{4}$ Department of Cardiac Imaging, Barts Heart Centre, \\ Barts Health NHS Trust, London, UK; ${ }^{5}$ Barts Cardiovascular Clinical Trials Unit, Queen Mary University of London, London, UK \\ \#These authors contributed equally to this work. \\ Correspondence to: Dr. Daniel A. Jones. Department of Cardiology, Barts Heart Centre, St. Bartholomew's Hospital, West Smithfield, London EC1A \\ 7BE, UK. Email: daniel.jones@qmul.ac.uk.
}

\begin{abstract}
Background: Patients with ischaemic heart disease and previous coronary artery bypass grafting (CABG) often need coronary evaluation by means of invasive coronary angiography (ICA). ICA in such patients is technically more challenging and carries a higher risk of complications including kidney damage, myocardial infarction, stroke and death. Improvements in Computed Tomography Cardiac Angiography (CTCA) technology have ensured its emergence as a useful clinical tool in CABG assessment, allowing for its potential use in planning interventional procedures in this patient group.

Methods: The BYPASS-CTCA study is a prospective, single centre, randomised controlled trial assessing the value of upfront CTCA in patients with previous surgical revascularisation undergoing ICA procedures. A total of 688 patients with previous CABG, requiring ICA for standard indications, will be recruited and randomised to receive ICA alone, or CTCA prior to angiography. Subjects will be followed up over a 12-month period post procedure. The primary endpoints are ICA procedural duration, incidence of contrast-induced nephropathy (CIN) and patient satisfaction scores post ICA. Secondary endpoints include contrast dose $(\mathrm{mL})$ and radiation dose $(\mathrm{mSv})$ during ICA, number of catheters used, angiography-related complications and cost-effectiveness of CTCA (QALY) over 12 months.

Discussion: The study will investigate the hypothesis that CTCA prior to ICA in patients with previous CABG can reduce procedural duration, post-procedural kidney damage and improve patient satisfaction, therefore strengthening its role in this group of patients.
\end{abstract}

Trial Registration: The study is registered on ClinicalTrials.gov which is a resource maintained by the U.S. National Library of Medicine. Registration number NCT03736018.

Keywords: Ischaemic heart disease; coronary artery bypass grafting (CABG); invasive coronary angiography (ICA); Computed Tomography Cardiac Angiography (CTCA); contrast-induced nephropathy (CIN)

Submitted Apr 11, 2021. Accepted for publication Jun 17, 2021.

doi: $10.21037 /$ atm-21-1455

View this article at: https://dx.doi.org/10.21037/atm-21-1455 


\section{Introduction}

Each year, approximately 300,000 coronary artery bypass graft (CABG) surgeries are performed in the US (1) and 20,000 in the UK (2). The purpose of CABG is to restore an adequate blood supply to blood deprived myocardium, and the success of the operation depends mainly on the patency of the bypass grafts (3). Indeed, the natural history of bypass grafts is such, that $10-15 \%$ of venous grafts become occluded per year, with $50 \%$ occluded after a period of 10 years (4-6). These patients therefore often need coronary evaluation by means of invasive coronary angiography (ICA) in order to explore recurrence of chest pain, heart failure, or for pre-operative evaluation prior to cardiac valve replacement or high-risk non-cardiac surgery.

While ICA remains the gold standard for both coronary artery and graft evaluation, it is still associated with a risk of complications such as kidney damage, myocardial infarction, stroke, vascular complications and death $(7,8)$. The risk of these complications is significantly increased in the CABG population, in part due to increased co-morbidity. Cardiac catheter manipulation to engage the grafts is associated with an increased risk of embolic stroke or dissection (9), as graft engagement is technically more difficult than native vessels, with the location of the bypass ostia often variable. ICA in CABG patients is often performed via the femoral route, lasts longer, and leads to higher irradiation and nephrotoxicity than ICA in patients without CABG $(10,11)$. This emphasises the need for improvement in techniques to aid the search for bypass grafts during ICA, a topic that has been well discussed for many years.

Improvements in Computed Tomography Cardiac Angiography (CTCA) technology have allowed its emergence as a useful clinical tool in CABG assessment. CTCA allows detailed evaluation of venous and arterial grafts, and is also highly accurate at detecting stenoses in bypass grafts (see Figure 1) with sensitivity, specificity, negative and positive predictive values of $97 \%, 97 \%, 93 \%$ and $99 \%$, respectively (12-14). The evolution of scanners has resulted in markedly reduced radiation doses $(<2 \mathrm{mSv})$, required contrast media $(<90 \mathrm{~mL})$ and acquisition time (seconds). Reconstruction of any structure of interest in 3D by CTCA means it is now possible to fuse reconstructions in real time with other forms of interventional imaging such as angiography $(15,16)$. These images can be used to guide interventional procedures, facilitate identification of the origin of the grafts and indicate the appropriate catheters for graft selection. CTCA itself is well tolerated with previous studies demonstrating high patient satisfaction scores (17). These factors, along with the low risk of complications, means that CTCA has the potential to optimise ICA in this patient group.

Observational data exists to support the utility of CTCA in guiding cardiologists performing ICA in CABG patients to assist graft detection. Two studies, one using pre-existing CT scans (18) and one dedicated CTCAs (19), have found that prior CTCA before ICA could lead to a reduction in procedural time, contrast administered and ionizing radiation exposure during ICA however, no randomised study has been performed to date to assess whether routine CTCA prior to planned ICA improves procedure metrics or patient outcomes. We present the protocol of the BYPASSCTCA study in accordance with the SPIRIT reporting checklist (available at https://dx.doi.org/10.21037/atm-211455).

\section{Methods}

\section{Hypothesis}

CTCA prior to ICA, in patients who have had previous CABG, leads to reduced procedure duration, postprocedural kidney damage and improved patient satisfaction, compared to ICA alone.

\section{Trial design}

The BYPASS-CTCA study is a prospective, single centre, randomised controlled trial to determine the value of CTCA in improving patient satisfaction and reducing iodinated contrast load, procedural duration and complications in patients who have had previous CABG undergoing ICA +/- percutaneous coronary intervention (PCI). The study is registered on Clinicaltrials.gov, number NCT03736018 (20). The trial will be conducted in accordance with the Declaration of Helsinki (as revised in 2013) and was approved by the local ethics committee (London - Westminster Research Ethics Committee, reference 18/LO/1583).

\section{Study population}

Patients [688] with previous CABG undergoing invasive coronary procedures involving the administration of intracoronary iodinated contrast media (ICA +/- PCI), over the age of 18 and able to give written informed consent, will be recruited at The Barts Heart Centre (BHC), based at St Bartholomew's Hospital, London UK. This is the largest cardiac centre in the UK, serving a 

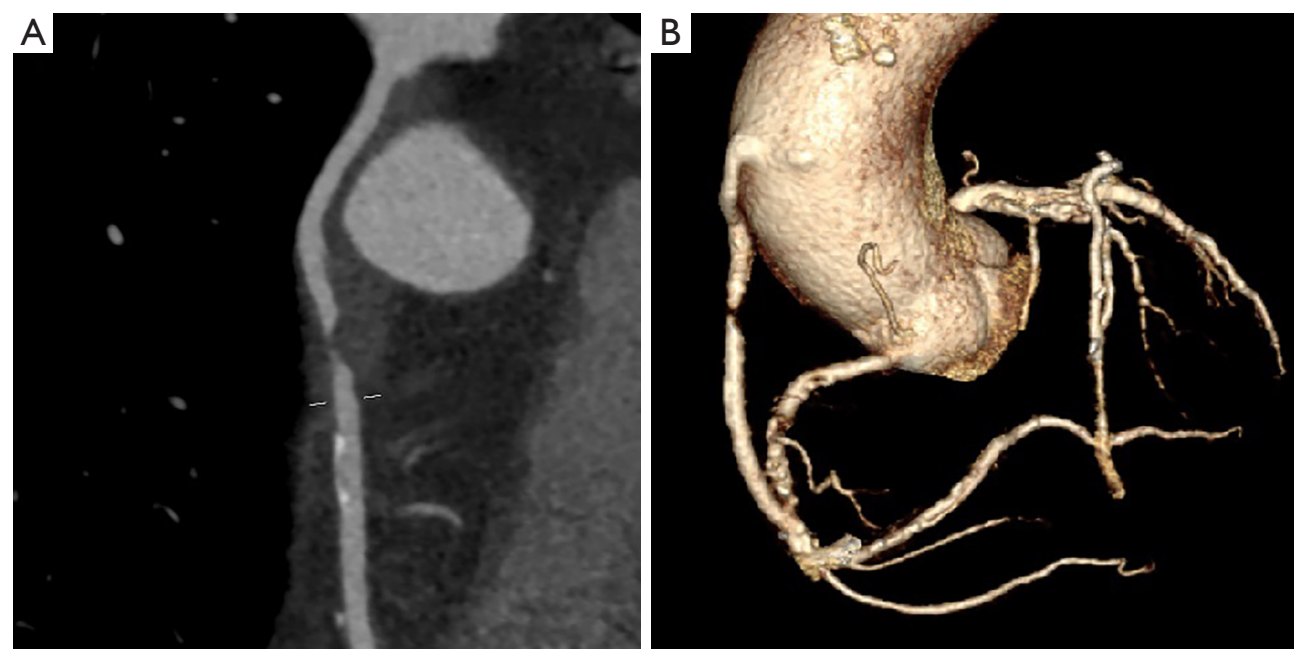

Figure 1 Reconstructed computed tomography (CT) images showing (A) severe stenosis in a saphenous vein graft to the right coronary artery in 2D, and (B) severe stenosis in the same vessel (saphenous vein graft to right coronary artery) reconstructed in $3 \mathrm{D}$.

population of approximately 6 million people from North East and Central London and is a 24/7 centre performing approximately 6,000 angiograms and 2,000 non-primary angioplasties per year. Patients fulfilling inclusion criteria at the time of arrival to BHC will be screened by the research doctor or principal investigator (PI) for entry into the study. Patients may also be referred by their attending physician to the research team once a decision for elective ICA has been made. Patients will be given time to consider their full participation, although patients with an acute coronary syndrome (ACS) will, by the urgent nature of their condition, have less than 24 hours to consider participation. See Table 1 for full inclusion and exclusion criteria.

\section{Intervention-CTCA}

In elective planned ICA cases, the aim is to perform the CTCA at least 2 weeks prior to the ICA in order to ensure adequate time between the two investigations, however patient preference will be taken into account meaning that in some cases CTCA may be performed on the same day as the ICA. In patients presenting with ACS, the CTCA and ICA will also be performed within 24 hours, based on guidelines and clinical pathways.

All CTCAs will be performed at BHC using a thirdgeneration dual source CT scanner (Somatom FORCE, Siemens, Germany). The scan range will include the ascending aorta from the Sinuses of Valsalva to the Subclavian arteries to visualize the origins of the grafts. Prospectively
ECG-triggered high-pitch spiral scanning or sequential scanning protocols will be used, depending on patients' characteristics and heart rate. The scan time required for the defined anatomical range will be $<5 \mathrm{~s}$. Automated exposure control (CARE Dose 4D) will be used. It is expected that radiation exposure to patients will be in the range of 0.4-8 mSv, accounting for variability related to patient's weight, body mass index (BMI) and habitus. Radiation dose and contrast volume administered during CTCA will be recorded for those patients in the CT arm. A set of $0.75 \mathrm{~mm}-$ thick images will be reconstructed with a reconstruction increment of $0.5 \mathrm{~mm}$ using an iterative reconstruction algorithm (ADMIRE, strength 2). The dataset will be postprocessed according to multiplanar reconstruction (MPR's) and maximum intensity projections (MIP's). Fluoroscopic views that optimize the visualization of the graft origins for cannulation will be identified, the angles will be communicated to the interventional team (LAO/RAO, Cra/ $\mathrm{Cau}$ ) and corresponding 2D images will be generated from the CTCA data and displayed on the monitor during ICA to facilitate graft engagement. Additionally, the CT images of each patient will be post-processed to reconstruct a $3 \mathrm{D}$ volume of the aortic arch, subclavian arteries and proximal portion of the grafts.

\section{Invasive coronary angiogram procedure}

All coronary angiograms will be performed at BHC. X-ray exposure settings (low dose with a default frame rate of 4 
Table 1 Inclusion and exclusion criteria for participation in BYPASS-CTCA

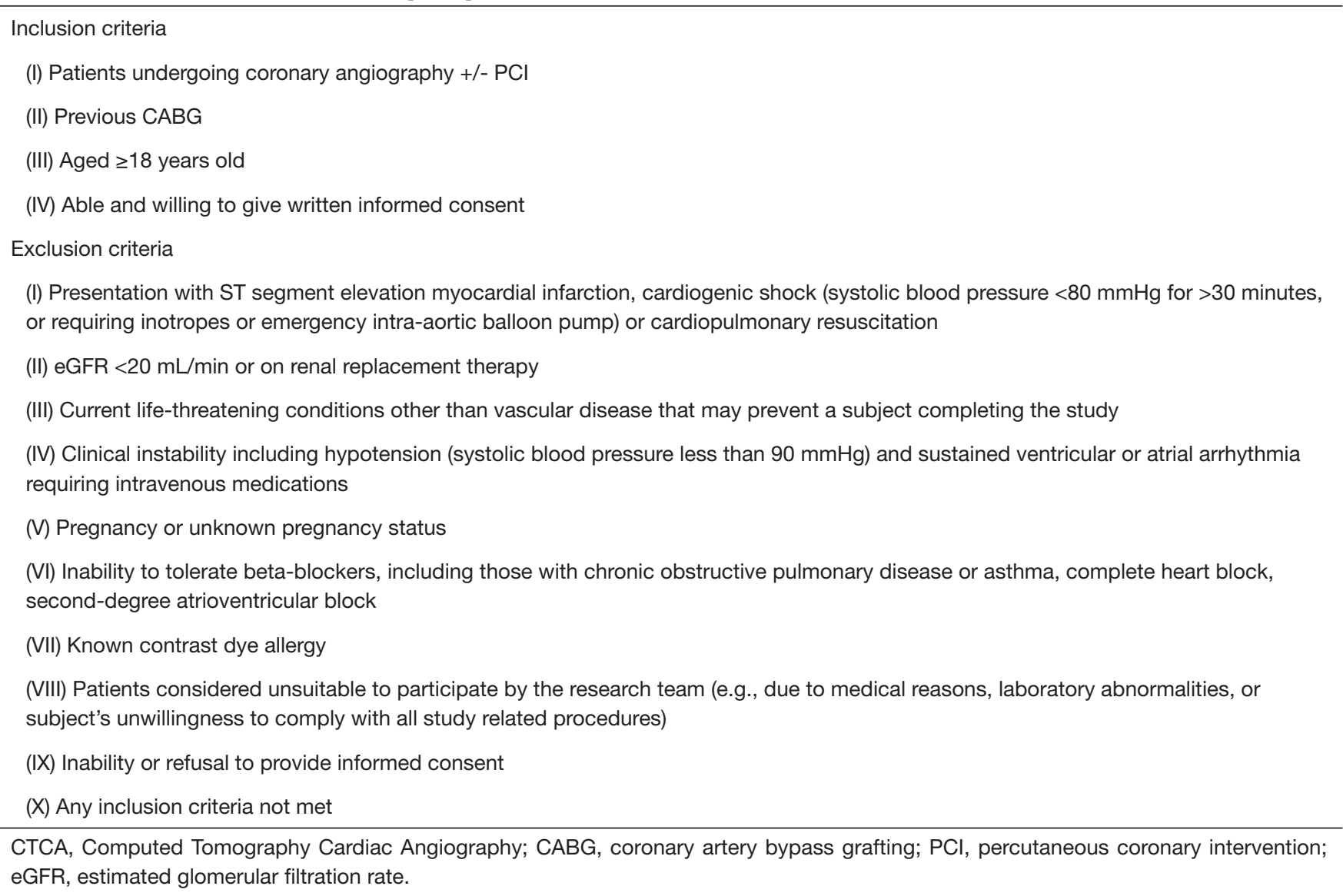

frames/s fluoroscopy and 7.5 frames/s acquisition), radiation protection and contrast agents used will be the same in all studied patients. To minimize the effect of the operators on outcomes all the ICAs will be performed by experienced cardiologists. Femoral or radial access will be chosen based on the interventional cardiologist's decision. In patients randomised to CTCA, the CTCA report detailing the number of grafts, location, and patency will be made available to the operator at the time of the ICA. When possible, reconstructed $3 \mathrm{D}$ images will also be initially registered and overlaid over the real-time fluoroscopic images using image fusion software.

\section{Randomisation}

Eligible patients that have given written informed consent will be block randomised on a 1:1 basis (using an online randomisation database) to receive ICA alone, or CTCA prior to angiography. Since ACS patients are at a higher risk of developing CIN, these patients will be included with equal stratification in both groups.

\section{Study endpoints}

The primary endpoint for the study is a composite endpoint consisting of:

(I) ICA procedure duration;

(II) Incidence of CIN, as defined by the Kidney Disease Improving Global Outcomes (KDIGO) criteria (21);

(III) Patient satisfaction scores post ICA (based on a validated questionnaire).

The secondary endpoints are:

(I) Contrast amount (mL) administered during ICA;

(II) Radiation exposure ( $\mathrm{mSv}$ ) during ICA;

(III) Angiography related complications (coronary or aortic dissection, stroke, bleeding and vascular access complications); 
(IV) Radial access rates during ICA;

(V) Number of catheters used during ICA;

(VI) Number of grafts not identified during ICA;

(VII) Accuracy of the CTCA for detecting graft patency;

(VIII) Cost effectiveness of CTCA (QALY) measured over 12 months of follow-up.

\section{Participant timeline}

At baseline, a comprehensive medical history, medication history and physical examination (including height, weight, $\mathrm{BMI}$, resting $\mathrm{BP}$ and heart rate) will be recorded. Following consent and prior to their ICA procedure venous blood will be taken to measure baseline renal function or alternatively, any recent clinically relevant bloods will be utilised. The participant will be randomised to CTCA or not prior to their planned angiographic procedure. A Quality of Life assessment (EQ5D) and a Seattle Angina Questionnaire (SAQ) will be performed prior to angiography +/- PCI.

ICA procedure duration will be measured using a timer started after the insertion of the arterial catheter and stopped when the catheters are removed, after opacification of the native coronary arteries and grafts. As for all interventional radiology procedures, the parameters corresponding to the applied irradiation and quantity of contrast agent required will be reported in each group. Thus, the total air kerma (AK) in milliGray (mGy), dose area product (DAP) in centiGray per square centimeters $\left(\mathrm{cGy} / \mathrm{cm}^{2}\right)$, fluoroscopy time in minutes, total radiation time in minutes and amount of iodinated contrast agent in millilitres $(\mathrm{mL})$ will be reported.

After the procedure the patient will be asked to complete a patient satisfaction questionnaire (17). A blood test will be taken at 48-72 hours for renal function (samples up to 1 week post procedure will be accepted for the assessment of CIN as per the KDIGO criteria). The participant will be asked to come back at 3 months to repeat the renal function test. Quality of life assessments (EQ5D and SAQ) will be repeated at 3 months post procedure and an assessment of major adverse cardiac events (MACE) will be carried out. At 1 year, participants will have telephone follow-up for assessment of MACE, EQ5D and SAQ. The duration of the study for each individual patient will be 1 year (see Figure 2).

\section{Patient satisfaction, cost and bealth outcome data}

Patient satisfaction with both the CTCA and the ICA will be assessed following each procedure using a validated questionnaire. Length of stay and MACE will be recorded from telephone contact with patients, hospital and primary care records, and deaths from the Central Registry Office or equivalent. EQ5D is a questionnaire, designed in Europe, which evaluates generic quality of life. The EQ5D descriptive system is a preference-based Health-related Quality of Life measure with one question for each of the five dimensions that include mobility, self-care, usual activities, pain/discomfort, and anxiety/depression. The Seattle Angina Questionnaire (SAQ) is a self-administered, disease-specific health status questionnaire that measures clinically important dimensions of health in patients with ischemic heart disease (22).

\section{Sample size}

The study is powered to detect a composite primary endpoint with individual power for all three primary study endpoints. The primary endpoint requiring the largest sample size is CIN; 510 patients give $80 \%$ power (significance level 0.05) to demonstrate a CIN reduction of $60 \%$, assuming an estimated CIN rate of $12 \%$. For the other endpoints 510 patients provides $90 \%$ power (significance level 0.05 ) to demonstrate a 3.5 minutes (14\%) reduction in procedure time, assuming a mean procedure time of 25 minutes (SD of 12 ) and $90 \%$ power (significance level 0.05 ) to demonstrate a 15\% improvement in patient satisfaction scores, assuming an estimated mean control score of $1.5 \pm 0.7$ (1.72 compared to 1.5 ) [data from previously published ICA satisfaction studies $(17,23,24)]$. We applied the Bonferroni correction and used $\mathrm{a}=0.017$ in the calculations. This gives a total sample size of $\mathrm{N}=618$ which increases to $\mathrm{N}=688$ after accounting for drop out, based on $80 \%$ power for the CIN effect, and $90 \%$ power for procedure duration and patient satisfaction correcting for multiple comparisons.

\section{Statistical analysis}

Analysis will be on an intention-to-treat basis. The total number of subjects and the number and proportion of subjects with CIN will be presented by treatment group and overall. The effect size will be presented as an odds ratio with confidence intervals obtained during logistic regression. For procedural duration and patient satisfaction scores, the total number of subjects with data, mean (SD) and median [IQR] will be presented by treatment group 


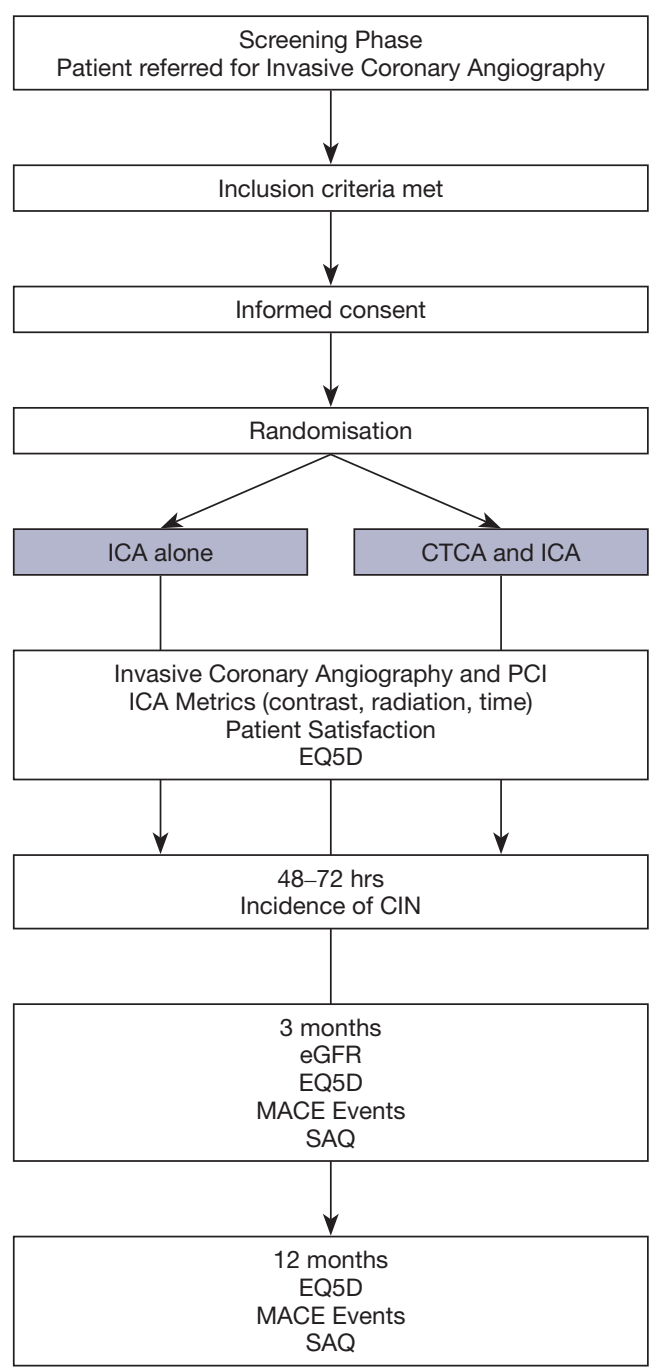

Figure 2 Study flowchart. CTCA, Computed Tomography Cardiac Angiography; EQ5D, Quality of Life Questionnaire; CIN, contrast-induced nephropathy; eGFR, estimated glomerular filtration rate; MACE, major adverse cardiac events; SAQ, Seattle Angina Questionnaire. CCS, Canadian Chest Pain Score, CTCA, Computed Tomography Coronary Angiography; CABG, coronary artery bypass grafts.

and overall. Differences will be assessed using a two-sample $t$-test with unequal variances and effect size presented as the difference between groups with $95 \%$ confidence intervals. If the data is considered non-normally distributed, a MannWhitney $U$ test will be conducted. For all three primary endpoints adjustment for ACS status and creatinine at baseline will be made. Subgroup analysis will be performed for primary outcomes stratified by ethnicity (white versus black, Asian and minority ethnic), type of presentation (stable/elective versus ACS), seniority of the clinician performing the ICA (consultant versus interventional trainee) and knowledge of graft anatomy [operation note/previous angiogram since CABG (yes versus no)]. Unadjusted effect sizes will be shown by subgroup and for all patients using a modified forest plot, along with a test for heterogeneity. For secondary endpoint analysis confidence intervals and statistical tests are of an exploratory nature.

\section{Discussion}

\section{Trial management}

This study is supported by the Barts Cardiovascular Clinical Trials Unit (CVCTU), a branch of the Barts CTU UKCRC Reg No. 4. Similar to other trials they support (25), the CVCTU will oversee the management and conduct of the trial, including safety reporting, co-ordination of trial committees, statistical analysis and reporting, and database management and electronic case report form (eCRF) design.

Source data will be collected by delegated members of the research team and captured in the eCRF using the REDCap database with electronic signatures and an audit trail. Information related to participants will be kept confidential and managed in accordance with the NHS Code of Confidentiality and the GDPR and UK Data Protection Act 2018. During the study, participant study records and paper source documents will by marked by a single pseudoanonymised subject identifier. This will be identified on eCRFs and any paper source documentation. All paper source data will be kept securely in participant study files or investigator site files within locked cabinets in restricted access rooms within St. Bartholomew's Hospital.

\section{Adverse events (AEs) reporting}

All AEs and serious AEs will be recorded and reported to the PI. Related unexpected serious AEs, as assessed by the PI, will be reported within 24 hours of the research team becoming aware, to the Joint Research Management Office and the main Research Ethics Committee, as per the local and CVCTU policy (25).

\section{Monitoring}

A Trial Steering Committee (TSC) composed of three 
independent experts in the fields of cardiac imaging (CT), and cardiology along with the investigators and a lay member will monitor the study. They will meet before patient recruitment and as per the TSC charter thereafter to assess trial conduct, recruitment, feasibility or other arising issues.

An independent Data Safety Monitoring Board (DSMB) will be formed to monitor patient safety. The DSMB will meet prior to initiation of the study, after recruitment of 10 patients, and thereafter as per the DSMB charter. If there is serious concern with the safety of the participants, the DSMB may recommend early termination of the study. An interim analysis for safety by treatment arm will be undertaken once 400 patients have been recruited and results reported to the DSMB.

\section{Trial status}

The trial opened to recruitment in November 2018, with an estimated recruitment period of 30 months and will finish 1 year after the last participant is recruited with an estimated end-date of late 2022/early 2023. All relevant data will be submitted to peer reviewed journals for publication. We aim to publish the primary endpoint results after the last recruited participant has reached their 3-month follow-up with secondary analyses assessing 1 year MACE outcomes and cost-effectiveness after the trial is completed.

\section{Limitations}

As this is a single centre study, limitations may include the application of results to other centres where CTCA may not be readily available. The interpretation and application of information from CTCA also currently still varies amongst clinicians however this study may provide further insight into this.

\section{Conclusions}

ICA remains the gold standard for evaluation of both native coronary arteries and grafts, however in patients with previous CABG it remains technically more challenging and associated with a higher risk of complications. The role of CTCA in these patients has been increasing with advancing technology. The BYPASSCTCA study aims to show that the use of CTCA in this patient population prior to ICA leads to improvements in patient satisfaction as well as a reduction in procedural time and post-procedure kidney problems. Results may influence future techniques to improve outcomes in this group of patients.

\section{Acknowledgments}

Funding: This work is supported by the National Institute of Health Research, Research for Patient Benefit award (PB-PG-1216-20028). DAJ is funded by The Barts Charity Cardiovascular Programme Award (G00913), KSR by a National Institute of Health Research Academic Clinical Lectureship.

\section{Footnote}

Reporting Checklist: The authors have completed the SPIRIT reporting checklist. Available at https://dx.doi. org/10.21037/atm-21-1455

Peer Review File: Available at https://dx.doi.org/10.21037/ atm-21-1455

Conflicts of Interest: All authors have completed the ICMJE uniform disclosure form (available at https:// dx.doi.org/10.21037/atm-21-1455). FP reports receiving institutional research support from Siemens Healthineers. The other authors have no conflicts of interest to declare.

Ethical Statement: The authors are accountable for all aspects of the work in ensuring that questions relating to the accuracy or integrity of any part of the work are appropriately investigated and resolved. The trial will be conducted in accordance with the Declaration of Helsinki (as revised in 2013). The study is approved by the local ethics committee (London - Westminster Research Ethics Committee, reference 18/LO/1583) and informed consent will be taken from all study participants.

Open Access Statement: This is an Open Access article distributed in accordance with the Creative Commons Attribution-NonCommercial-NoDerivs 4.0 International License (CC BY-NC-ND 4.0), which permits the noncommercial replication and distribution of the article with the strict proviso that no changes or edits are made and the original work is properly cited (including links to both the formal publication through the relevant DOI and the license). 
See: https://creativecommons.org/licenses/by-nc-nd/4.0/.

\section{References}

1. Epstein AJ, Polsky D, Yang F, et al. Coronary revascularization trends in the United States, 2001-2008. JAMA 2011;305:1769-76.

2. Ludman PF. BCIS audit returns adult interventional procedures. 2012.

3. Møller CH, Perko MJ, Lund JT, et al. Graft patency after off-pump versus on-pump coronary artery surgery in highrisk patients. Scand Cardiovasc J 2010;44:161-7.

4. Goldman S, Zadina K, Moritz T, et al. Long-term patency of saphenous vein and left internal mammary artery grafts after coronary artery bypass surgery: results from a Department of Veterans Affairs Cooperative Study. J Am Coll Cardiol 2004;44:2149-56.

5. Fitzgibbon GM, Kafka HP, Leach AJ, et al. Coronary bypass graft fate and patient outcome: angiographic followup of 5,065 grafts related to survival and reoperation in 1,388 patients during 25 years. J Am Coll Cardiol 1996;28:616-26.

6. Rodés-Cabau J, Facta A, Larose E, et al. Predictors of aorto-saphenous vein bypass narrowing late after coronary artery bypass grafting. Am J Cardiol 2007;100:640-5.

7. Scanlon PJ, Faxon DP, Audet AM, et al. ACC/AHA guidelines for coronary angiography. A report of the American College of Cardiology/American Heart Association Task Force on practice guidelines (Committee on Coronary Angiography). Developed in collaboration with the Society for Cardiac Angiography and Interventions. J Am Coll Cardiol 1999;33:1756-824.

8. Noto TJ Jr, Johnson LW, Krone R, et al. Cardiac catheterization 1990: a report of the Registry of the Society for Cardiac Angiography and Interventions (SCA\&I). Cathet Cardiovasc Diagn 1991;24:75-83.

9. Werner N, Bauer T, Hochadel M, et al. Incidence and clinical impact of stroke complicating percutaneous coronary intervention: results of the Euro heart survey percutaneous coronary interventions registry. Circ Cardiovasc Interv 2013;6:362-9.

10. Delewi R, Hoebers LP, Råmunddal T, et al. Clinical and procedural characteristics associated with higher radiation exposure during percutaneous coronary interventions and coronary angiography. Circ Cardiovasc Interv 2013;6:501-6.

11. Sanmartin M, Cuevas D, Moxica J, et al. Transradial cardiac catheterization in patients with coronary bypass grafts: feasibility analysis and comparison with transfemoral approach. Catheter Cardiovasc Interv 2006;67:580-4.

12. Hamon M, Lepage O, Malagutti P, et al. Diagnostic performance of 16- and 64-section spiral CT for coronary artery bypass graft assessment: meta-analysis. Radiology 2008;247:679-86.

13. Goetti R, Leschka S, Baumüller S, et al. Low dose highpitch spiral acquisition 128-slice dual-source computed tomography for the evaluation of coronary artery bypass graft patency. Invest Radiol 2010;45:324-30.

14. Meyer TS, Martinoff S, Hadamitzky M, et al. Improved noninvasive assessment of coronary artery bypass grafts with 64-slice computed tomographic angiography in an unselected patient population. J Am Coll Cardiol 2007;49:946-50.

15. Markelj P, Tomaževič D, Likar B, et al. A review of 3D/2D registration methods for image-guided interventions. Med Image Anal 2012;16:642-61.

16. Collet C, Sonck J, Leipsic J, et al. Implementing Coronary Computed Tomography Angiography in the Catheterization Laboratory. JACC Cardiovasc Imaging 2020. [Epub ahead of print]. doi: 10.1016/ j.jcmg.2020.07.048.

17. Feger $S$, Rief $M$, Zimmermann E, et al. Patient satisfaction with coronary CT angiography, myocardial CT perfusion, myocardial perfusion MRI, SPECT myocardial perfusion imaging and conventional coronary angiography. Eur Radiol 2015;25:2115-24.

18. Plessis J, Warin Fresse K, Cahouch Z, et al. Value of Image Fusion in Coronary Angiography for the Detection of Coronary Artery Bypass Grafts. J Am Heart Assoc 2016;5:002233.

19. Jones DA, Castle EV, Beirne AM, et al. Computed tomography cardiac angiography for planning invasive angiographic procedures in patients with previous coronary artery bypass grafting. EuroIntervention 2020;15:e1351-7.

20. Clinical Trials.gov [Internet] Randomised Controlled Trial to Assess Whether Computed Tomography Cardiac Angiography Can Improve Invasive Coronary Angiography in Bypass Surgery Patients. [Internet]. [cited 2021 Jun 11]. Available online: https://clinicaltrials.gov/ ct2/show/NCT03736018

21. Fliser D, Laville M, Covic A, et al. A European Renal Best Practice (ERBP) position statement on the Kidney Disease Improving Global Outcomes (KDIGO) clinical practice guidelines on acute kidney injury: part 1: definitions, conservative management and contrast-induced nephropathy. Nephrol Dial Transplant 2012;27:4263-72. 
22. Spertus JA, Winder JA, Dewhurst TA, et al. Development and evaluation of the Seattle Angina Questionnaire: a new functional status measure for coronary artery disease. J Am Coll Cardiol 1995;25:333-41.

23. Mangelsdorff AD. Patient satisfaction questionnaire. Med Care 1979; 17:86-90.

24. Rief M, Feger S, Martus P, et al. Acceptance of Combined Coronary CT Angiography and Myocardial CT Perfusion versus Conventional Coronary Angiography in Patients

Cite this article as: Beirne AM, Rathod KS, Castle E, Andiapen M, Richards A, Bellin A, Hammond V, Godec T, Moon JC, Davies C, Bourantas CV, Wragg A, Ahluwalia A, Pugliese F, Mathur A, Jones DA. The BYPASS-CTCA Study: the value of Computed Tomography Cardiac Angiography (CTCA) in improving patient-related outcomes in patients with previous bypass operation undergoing invasive coronary angiography: Study Protocol of a Randomised Controlled Trial. Ann Transl Med 2021;9(17):1395. doi: 10.21037/atm-21-1455 with Coronary Stents--Intraindividual Comparison. PLoS One 2015;10:e136737.

25. Beirne AM, Mitchelmore O, Palma S, et al. NITRATECIN Study: Protocol of a Randomized (1:1) SingleCenter, UK, Double-Blind Placebo-Controlled Trial Testing the Effect of Inorganic Nitrate on ContrastInduced Nephropathy in Patients Undergoing Coronary Angiography for Acute Coronary Syndromes. J Cardiovasc Pharmacol Ther 2021;26:303-9. 